\title{
Applying clustering to statistical analysis of student reasoning about two-dimensional kinematics
}

\author{
R. Padraic Springuel, Michael C. Wittmann, and John R. Thompson \\ Department of Physics and Astronomy, Center for Science and Mathematics Education Research, College of Education and Human \\ Development, University of Maine, Orono, Maine 04469, USA
}

(Received 24 May 2007; published 3 December 2007)

\begin{abstract}
We use clustering, an analysis method not presently common to the physics education research community, to group and characterize student responses to written questions about two-dimensional kinematics. Previously, clustering has been used to analyze multiple-choice data; we analyze free-response data that includes both sketches of vectors and written elements. The primary goal of this paper is to describe the methodology itself; we include a brief overview of relevant results.
\end{abstract}

DOI: 10.1103/PhysRevSTPER.3.020107

PACS number(s): 01.40.Fk, 01.40.gf

\section{INTRODUCTION}

In physics education research (PER), there is a long tradition of asking content-specific questions along the lines of "What do students know about Topic X?"1,2 Typically, a researcher answering such a question designs (or uses) a question about topic $\mathrm{X}$ that is supposed to elicit the ideas that students have. The researcher then administers this question (as a pretest, homework, exam question, interview, etc.) to a group of students. In most cases (especially when the data set is large) the researcher then takes this collection of student responses and classifies them according to the themes that are present in the students' answers. Two reasons exist for this classification of student responses. First, it allows researchers and teachers to have a finite number of categories to attend to, rather than attempting to understand every individual student's nuanced response. Second, the question "What do students know about Topic X?" typically does not have a unique answer, and grouping mostly similar responses allows researchers and teachers to understand trends in the classroom more easily.

There are inherent difficulties in the classification of student responses as it is typically done, however. A researcher's impression of the responses is often undefined and incomplete-there is an art to good analysis, as well as a science. As a result, the categories may tend to find those groups of students that the researcher is already looking for (confirmation bias) or those who exhibit such an unusual answer that it catches the researcher's eye and remains in their memory (excited memory trace). Good researchers train themselves to ignore the excited memory trace issue (especially when such responses are rare) and to look explicitly for evidence to counter their claims (a disconfirmation bias), but such techniques are not foolproof. These and other issues of bias in interpreting student results are documented in PER; ${ }^{3,4}$ in particular, the effects of a researcher's theoretical perspective limiting interpretation of results are discussed in more detail by Wittmann and Scherr. ${ }^{5}$ While many of the problems of interpretation and analysis can be mitigated through accepted, diligent research practices, such as methods that examine and test for interrater and intrarater reliability, such practices are rarely cited in the PER literature, indicating that the issue of bias in interpretation may be considered, but is not publicized. The larger problem, though, is that issues related to fundamental psychological issues (such as memory traces and biases based on past experience) can never be completely removed so long as a person is creating and classifying the responses.

In this paper, we describe one response to the problem of researcher bias and the difficulties of fairly interpreting student responses. Broadly speaking, we apply data mining techniques that are algorithm driven (and often computer performed) to find information (usually in the form of some sort of pattern) in a large body of data. Cluster analysis is used to find the "natural" groupings of the data, sorting elements so that similar ones are in the same cluster while different ones are in different clusters. Since this is the goal of the classification schemes developed by researchers in PER, one might expect that cluster analysis methods can be used to find the natural groupings of students without an a priori knowledge of what those groups are (as a person doing the sorting would need). In fact, to some extent, Montenegro, Aubrect, and Bao did that when they used the $k$-means algorithm to group students into two broad categories based on their answers to a series of agree/disagree statements. ${ }^{6,7}$ This paper expands on their work by examining the application of a different cluster analysis method (agglomerative hierarchical clustering) to a particular PER task (drawing arrows indicating the directions of velocity and acceleration in kinematics) and discusses the benefits, and drawbacks, of doing so.

We note that a cluster analysis does not preclude a later model analysis ${ }^{8}$ or a factor analysis of one's data. Instead, cluster analysis can serve as a precursor to either of those analysis types. The power of cluster analysis lies in the clusters arising from the data and possibly uncovering unexpected relationships between student responses. We give examples below.

\section{THE PROBLEM}

The data for this paper are drawn from student pretests which were administered to students in the introductory algebra-based physics courses at the University of Maine in the fall of $2003(N=117), 2004(N=86)$, and $2005(N=82)$, and at Arizona State University during the spring of 2006 $(N=100)$. Pretests were administered after students had received standard lecture instruction on two-dimensional (2D) 


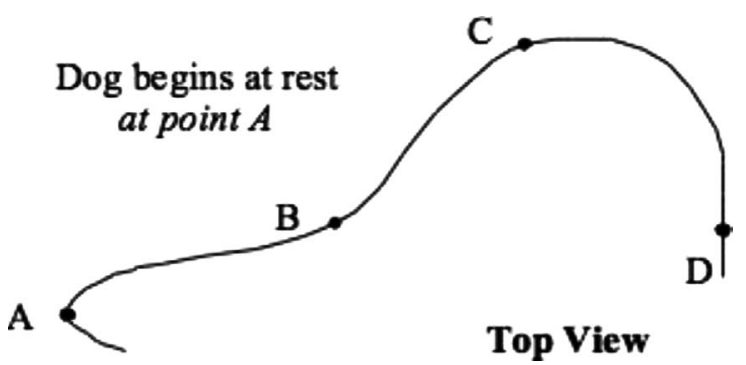

FIG. 1. A typical 2D kinematics question. In a horizontal plane, a dog starts at point $A$, speeds up until a point between $B$ and $C$ (not indicated on this diagram, but explicit on some question versions), and runs at constant speed past points $C$ and $D$. Students must draw a vector in the direction of the velocity at all points $A-D$. On a separate, identical diagram, they are asked to draw vectors in the direction of the acceleration. Based on similar questions asked at the University of Washington.

kinematics but before any tutorials on this subject. While there were some variations in the problems administered during different years, the basic task (which was based on similar questions asked at the University of Washington ${ }^{9}$ ) provided students with two copies of a diagram showing the trajectory an object was following as it moved with either increasing or constant speed. On these diagrams, four points were marked for the students to consider. On the first copy of the diagram, students were asked to draw vectors that represented the direction of the velocity at each of the marked points. On the second copy of the diagram they had to draw vectors that represented the direction of the acceleration at each of the marked points (see Fig. 1). Students were also asked to explain their reasoning for why they drew what they did.

A correct answer to this question is quite difficult in its details but we summarize ideas that one can use to arrive at a correct answer. We focus only on points $B$ and $C$ in the figure, since they are the ones that we analyze in depth in this paper. For point $B$, since one has both an increase in speed and a change in direction, the acceleration must contain a component due to each. Thus there will be a tangential component and a centripetal (pointing inward to the curve) component to the vector. For point $C$, there is no longer a tangential component, since the object is not speeding up in the direction of motion, but the centripetal component (pointing inward to the curve, and opposite to the direction of the centripetal component at $B$ ) remains. Similar reasoning can be used to determine the answers at $D$ and at $A$, though with the additional complexities of starting from rest at $A$ and moving in a straight line at $D$.

Variations on the 2D kinematics problem shown in Fig. 1 included flipping the diagram vertically and changing the context of the problem from a dog running on a flat track to a block sliding down an inclined plane. Similar problems with other variations have been analyzed by other researchers. ${ }^{9-11}$ The results of this research have been, in general, consistent in painting a stable pattern of the kinds of responses that students tend to make. Results, those of Thompson et al. ${ }^{10,11}$ in particular, have also shown that there are variations which may be context dependent. We do not explore these issues in this paper. Instead, we demonstrate the viability of the cluster analysis methodology in this paper and reserve the discussion of previous work in this field for a subsequent presentation in which we will explore the validity of the results obtained by cluster analysis by comparing them to said previous work.

\section{ANALYSIS}

Our goal was to describe student responses in such a way that a computer algorithm could cluster responses appropriately and group them reasonably. We describe the methods by which data were encoded from student responses and describe how we analyzed results in a way that allowed us to find groupings of student responses. In the next section, we discuss the groupings in more detail.

\section{A. Data encoding}

Since the actual clustering in this study was done by a computer, the first task was to take the wide variety of student responses that were given and convert them into a form that a computer could handle. In doing so, however, it was important to avoid pregrouping the responses in categories in much the same way as a researcher would if they were not planning on doing cluster analysis. To this end, the arrows at each point of a student response were described using categories that had as little interpretation involved in their definition as possible. These categories are listed in Table I along with a short description and picture of arrows that would fit the category. We used this encoding to describe both the velocity and the acceleration diagrams, but in this paper, we discuss results only from the acceleration diagrams.

For each student response, the student was given a 0 in categories which did not describe their answer and a 1 in categories which did describe their answer. It should be noted that the categories were not necessarily exclusive and, even in cases where there is an implied exclusivity, a student might still end up with a 1 in both categories if they drew more than one arrow at the point in question (about 3.8\% of point $B$ responses and $2.6 \%$ of point $C$ responses had this property). Such responses would probably have been ignored in another kind of analysis, but the point of this encoding was to be as completely descriptive as possible. Also, this practice has the result of placing those responses that did have multiple arrows between responses that only had one or the other in a triangular fashion [i.e., in the same way that $(1,1)$ can be considered between $(0,1)$ and $(1,0)$ because it is closer to either than they are to each other even though it does not fall on the line connecting $(1,0)$ and $(0,1)]$.

For the purposes of this study, the acceleration arrow at only two points of the trajectory were examined: the point where the object was speeding up as it followed a curved trajectory and the point where the object was moving with constant speed as it followed a curved trajectory (points $B$ and $C$ in Fig. 1, respectively). The result of this categorization was a 24-dimension (12 dimensions for each point) binary answer vector for each student [referred to as a student answer vector (SAV) in the subsequent discussion in order to 
TABLE I. Vector encoding. Sketched vectors were interpreted based on general criteria. Responses of "I don't know" were blanket responses encoded in the Reason codes (not presented in this paper).

\begin{tabular}{|c|c|c|}
\hline Category & Examples & Arrow description \\
\hline Curve following & & $\begin{array}{l}\text { Either one long arrow or a series of short ones that } \\
\text { tracked alongside the trajectory. }\end{array}$ \\
\hline Connect the dots & i & $\begin{array}{l}\text { Arrows that pointed directly to the next or previous } \\
\text { marked point along the motion. Such arrows were not coded } \\
\text { according to components. }\end{array}$ \\
\hline Head on point & & $\begin{array}{l}\text { An arrowhead on the trajectory at or near the point with } \\
\text { no body. }\end{array}$ \\
\hline Tangent & & Arrows that had a component tangent to the trajectory. ${ }^{\mathrm{a}}$ \\
\hline Back arrow & & $\begin{array}{l}\text { Arrows that generally pointed opposite the direction of } \\
\text { motion (note that this category necessarily modifies one of the } \\
\text { above). }\end{array}$ \\
\hline Centripetal & & Arrows that had a component centripetal to the trajectory. ${ }^{a}$ \\
\hline Centrifugal & & Arrows that had a component centrifugal to the trajectory. ${ }^{\text {a }}$ \\
\hline 0 arrow & & $\begin{array}{l}\text { Marks, sometimes text, that indicated a } 0 \text { magnitude } \\
\text { vector. }\end{array}$ \\
\hline No arrow & & $\begin{array}{l}\text { Nothing drawn at that point; used instead of "blank" to } \\
\text { emphasize that for some students this is a purposeful } \\
\text { behavior. }\end{array}$ \\
\hline Velocity label & & $\begin{array}{l}\text { A label, usually } v \text {, that identified the arrow as a velocity } \\
\text { vector }^{\text {b }}\end{array}$ \\
\hline Acceleration label & & $\begin{array}{l}\text { A label, usually } a \text {, that identified the arrow as an } \\
\text { acceleration vector. }\end{array}$ \\
\hline Indeterminate label & $\mathbb{1}$ & $\begin{array}{l}\text { A label that did not specify the type of quantity the arrow } \\
\text { was supposed to represent. }\end{array}$ \\
\hline
\end{tabular}

avoid confusion with the vectors students drew in their answers]. Once encoded in this fashion, each SAV had an average of 2.54 (standard deviation 0.83 ) nonzero dimensions.

We also found that some students did not draw arrows, or drew arrows in such a way that when their answer is taken as a whole (i.e., all four points on a diagram) their interpretation was not related to a physical direction. These alternative representations included position, velocity, and acceleration graphs as well as speedometers and accelerometers. Students who used these alternative representations, as well as students who always pointed their vectors in the same direction, regardless of the shape of the track, were excluded from the subsequent cluster analysis on the basis that any similarity their responses had with those students who followed the directions and had arrows indicating the direction of acceleration was coincidental because these groups were not performing the same task. As a result of this exclusion, 313 student responses were used in the cluster analysis. (We note that we carried out the cluster analysis described below using a data set that included alternative representation students, and we found that their responses naturally fall out of the analysis when they are marked as performing a different task, but "stick" to the response they share the most superficial resemblance to when they are not so marked. However, to clarify the process of cluster analysis, we leave them out of the data set for the rest of this paper.)

\section{B. Clustering method selection}

Once the data were in a form that could be handled by a computer program designed to perform a cluster analysis, it was still necessary to select the type of clustering algorithm to use. Below we discuss what some of the different possibilities are in the context of why we chose the method we did. This discussion is necessarily brief and incomplete. A more complete discussion of clustering algorithms can be found elsewhere. ${ }^{12,13}$

\section{Response and Cluster Distance}

Clustering algorithms are based on putting objects that are close together (i.e., have a small distance between them) into 


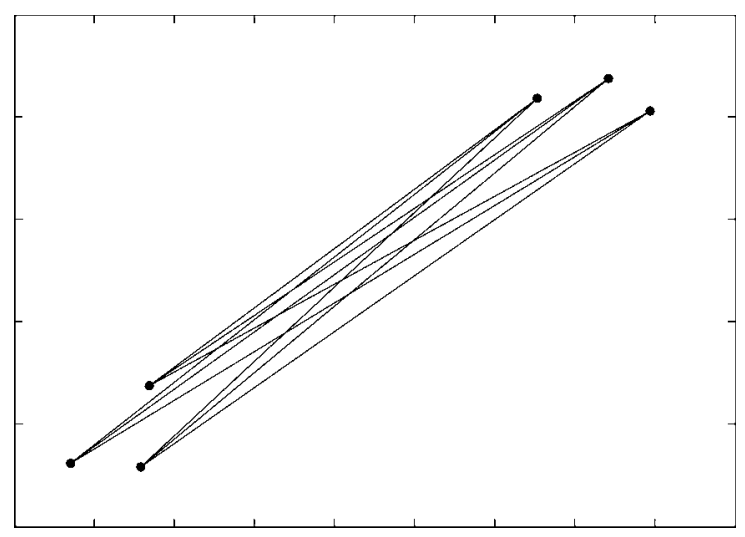

FIG. 2. A 2D example using an arbitrary coordinate system of the pairwise distances that would be averaged to find the distance between two clusters. In this example, the three points in the lower left are one cluster and the three in the upper right are the other. The distance between the clusters is the average length of the lines.

the same cluster while objects that are far apart (i.e., have a large distance between them) are in different clusters. Thus, one must choose how to define the distance between two objects (be they clusters or SAVs). While there are several different ways that distance between two vectors could be measured, it was decided to use the unweighted, normalized Euclidian distance between their heads for this study. This measure was chosen primarily for three reasons: (1) it is calculationally equivalent to the distance measure used by Montenegro, Aubrecht, and $\mathrm{Bao}^{6}{ }^{6}(2)$ it is readily available in the software package selected for this study; and (3) it is easy to compute by hand if necessary to check the computer calculations, as any distance between two student responses will be the square root of an integer divided by the number of dimensions in the answer vector.

The use of the unweighted, normalized Euclidian distance was not meant to imply anything about the nature of the student answer space. ${ }^{14}$

In defining how distance is measured, we must also define how the distance between groups of responses, not just single responses, is measured. Again, there are several available options; in this experiment the average-link method was selected. An average-link distance between two groups defines the distance between the groups as the average of all the pairwise distances between the individual elements of each group (see Fig. 2 for a two-dimensional example). This was chosen over single-link (the shortest of these pairwise distances) and maximum-link (the longest pairwise distance) methods. These two methods did not give as smooth a progression of distances for our binary data. We also did not choose the centroid-link method (distance between the average of each cluster) because the joining of two groups in that method can result in a combined group that is closer to another group than either of the two groups was separately.

\section{Membership and completeness}

After choosing how to define distance, one must decide whether each data point should be clustered and to how many groups it is allowed to belong. Possibilities include single- and multiple-cluster membership. For the sake of simplicity, we decided to cluster all data points (except for the alternative representations excluded earlier) and to restrict each data point to membership in a single cluster.

These choices made it easier to use preexisting software to perform the cluster analysis, but also created some problems. First, students whose answer could be considered noise (i.e., their responses are significantly different from all other responses) are forcibly grouped with other students to whom they are the closest, resulting in added uncertainty in the interpretation of clusters. Second, students do not necessarily have one and only one idea in their head when working on a problem. Instead they might have several competing ideas, which each manifest themselves at different points in their answer. By forcing each student response into one and only one group, we lose any chance we might otherwise have of seeing this phenomenon.

Despite these issues, it was decided that ease of use would be the first priority in this preliminary study and that dealing with these issues (and the possible development of software that would require) would be left to a later date.

\section{Algorithm}

Depending on how the previous quantities of distance, membership, and completeness have been chosen, one can choose an algorithm that carries out the clustering of student responses in the $n$-dimensional vector space. Two algorithms were available in the software package used in this study: $k$-means and agglomerative hierarchical clustering. Agglomerative hierarchical clustering starts by assigning each student response to its own cluster and then iteratively joining the two closest clusters until only one cluster remains. By maintaining records of each joining, the process can thus be used to extract any number of clusters from one to the size of the data set and shows how close these clusters are to each other. $k$-means, the algorithm used by Montenegro, Aubrecht, and $\mathrm{Bao},{ }^{7}$ divides data into clusters, but does not provide any information on how those clusters are related. It also requires the researcher to preselect the number of clusters in the data (though one can get around this by running the algorithm several times for different numbers of clusters and seeing which works the best). Finally, it also requires that one somehow guess (usually by random selection) where the center of each cluster is at the beginning, and is deterministic only for a particular set of initial guesses. Though Montenegro, Aubrecht, and Bao have had some success in dealing with these issues directly, ${ }^{7}$ it was decided that they would be avoided outright in this study by using agglomerative hierarchical clustering. This choice was not without its own issues, however, as will be discussed later in this paper.

Once the clustering method was chosen, running the data through the software that was obtained to do the clustering (the C clustering library, used through its PyCluster interface with Python) was a relatively simple exercise in computer programming (see the Appendix) the result of which is shown in Fig. 3. On this figure the vertical axis represents the distance between two clusters when they are joined and the horizontal axis shows student identification numbers (as- 


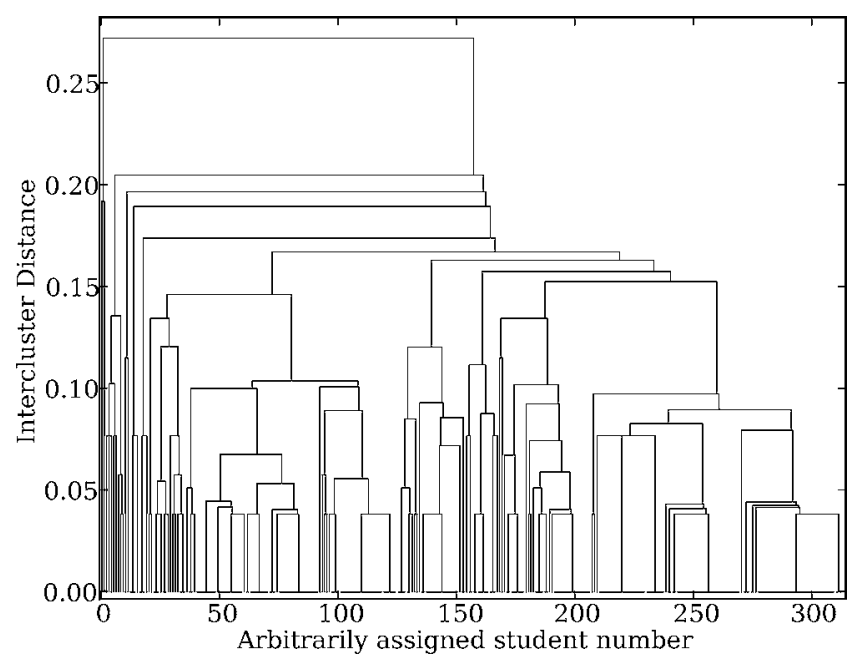

FIG. 3. Cluster tree of student results.

signed after the clustering was complete so that no line crossings were necessary in the figure). Furthermore, vertical lines represent individual clusters and horizontal lines represent the joining of the two clusters. To place these lines, vertical lines are always placed in the center of the group of students in that cluster and horizontal lines are placed at the height which corresponds to the distance between the two clusters that they join. Both horizontal and vertical lines end when they meet another line in order to keep the diagram free of clutter.

Without analyzing the content of the cluster tree yet, several interpretations of the figure itself are noteworthy. Though the clustering algorithm works by bringing together closest neighbors into a cluster (i.e., from the bottom up), we first describe the cluster tree from the top down, as if clusters are splitting apart. Also, it is easier to analyze the tree after we have removed all clusters with fewer than ten students, as in Fig. 4. We drop these clusters based on an ad hoc definition that 10 of 313 students is roughly 1 in 30; in a class of 30 students, you are unlikely to have a student with one of these responses. ${ }^{15}$ Our goal is to represent the most common clusters that one would observe in a given data set.

All student responses come together into a single cluster, located at the center and top of the figure. As one "travels down" the line, little jags indicate that outlier responses (with fewer than ten in each cluster) are branching off. Major branching begins at a cluster distance of about 0.17 . The cluster distance is not an important measure for our purposes, but it is noteworthy that five large clusters appear quickly. Some of these further split into several different clusters as the clusters get smaller and the distance between them also gets smaller. We describe the major clusters (i.e., the ones we consider meaningful) in the next section.

\section{INTERPRETATION OF CLUSTERS}

Whereas the computer has an easy time generating the clustering solution shown in the figure, it is left to researchers to make sense of the results. The post hoc analysis still

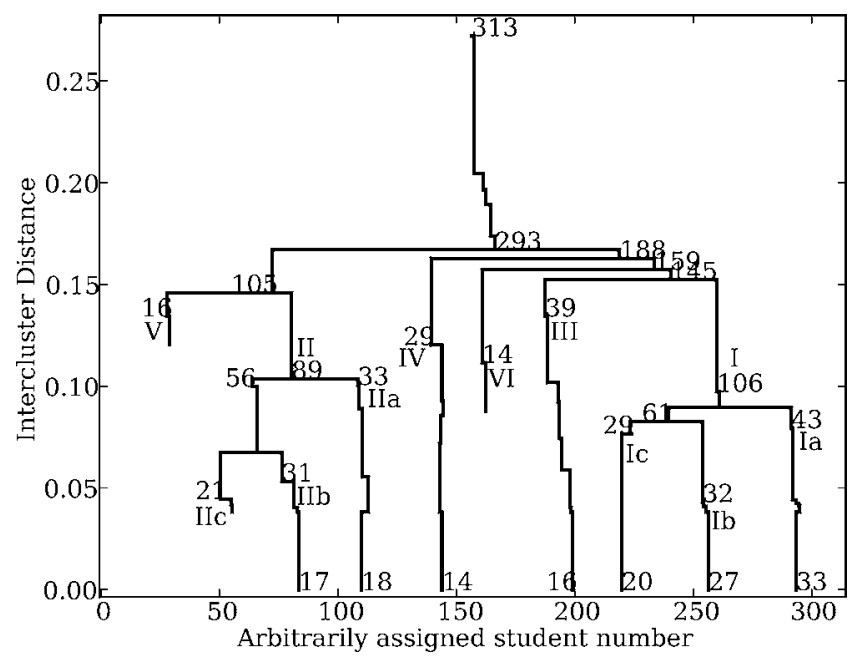

FIG. 4. The result of removing all clusters with fewer than ten students from the cluster tree. In this diagram, the number at each intersection is the number of students in the cluster created by that intersection. The roman numerals correspond to the groups in Table II.

requires researchers to interpret results, but the task is a fundamentally different one from finding categories. As a first step (and the only one we are ready to take in this preliminary study), the task is to describe each of the algorithmdetermined groupings and determine if the groupings are reasonable.

As noted above, we dropped all clusters in which there were ten or fewer people. This left us with a fairly sparse tree on which we could identify six major clusters, two of which had significant subclusters (see Fig. 4). To describe each of these clusters we looked for characteristics shared by all members of the cluster (defined as dominant characteristics) and those shared by the vast majority (75\% or higher) of the cluster (defined as prominent characteristics). The strength of this analysis is that we still have not determined what the students are thinking, but instead are focused on what they are doing. We consider this a strength because it allows us to carry out a different kind of research. Rather than seeking a model of student responses here, and then confirming it in, for example, an interview setting, we seek to find common response types and explore those more deeply in an interview setting. By using follow-up interviews, we can explore and document additional responses that give more insight into student reasoning. The clustering helps us find the student responses that occur most frequently and thus are most worth exploring further.

In the following discussion, the groups are discussed in order of decreasing size.

\section{A. Defining individual clusters}

\section{Group I: No C arrow $(n=106)$}

The first and largest group contained those students who had no acceleration arrow for an object that was moving around a curve at constant speed (no arrow at point $C$ on Fig. 
1) as a dominant characteristic for the group.

Perhaps these students were thinking that no change in speed meant there was no acceleration. Perhaps, related, they did not see a change in direction as due to an acceleration. ${ }^{9}$ And perhaps these students simply did not understand acceleration vectors. Each of these possibilities, and many others, could be considered.

The data also show that this group contained three distinct subgroups which were differentiated by their arrow type at the point where the object was speeding up as it went around the curve (point $B$ on Fig. 1): (Ia) tangent no arrow: those who had tangent arrows $(n=43)$, (Ib) $C t D$ no arrow: those who had connect-the-dots arrows $(n=32)$, and (Ic) Blanks: those who had no arrow $(n=29)$.

The discrepancy in the sum of the number of students in the three subgroups tangent no arrow, CtD no arrow, and blanks and the number of students in the main group no $C$ arrow is because of three students who are split out separately from the three subgroups. This group is not discussed because they fall below the ten-student cutoff threshold.

In summary, the students in each subgroup showed different dominant characteristics (in addition to the one they all had in common as members of no $C$ arrow). Either they do not indicate that changes in direction are a form of acceleration (tangent no arrow), they use a connect-the-dots approach but stop connecting dots at $C$ when the dog moves at constant speed (CtD no arrow), or they do not draw vectors at points $B$ and $C$ at all (blanks).

\section{Group II: Components $(n=89)$}

Students in the second largest cluster were those that used a mix of tangent and centripetal components at both points. This group includes those students answering the question correctly.

Unlike in no $C$ arrow, where the defining characteristic was a dominant characteristic, here the defining characteristics are only prominent characteristics. This is due to the fact that each of the subgroups had a distinct and independent mix of components, thereby preventing the full group from sharing a single dominant characteristic. These subgroups are as follows: (IIa) correct or circular: those with centripetal components at both points, some mixed with tangent ones at one or both points $(n=33)$; (IIb) pure tangent: those with just a tangent component at both points $(n=31)$, and (IIc) correct and tangent: those with both a tangent and centripetal component for changing speed, but only a tangent component for constant speed $(n=21)$.

Again, as with no $C$ arrow and its subgroups, some students are in below-threshold groups and the sum of students in the main subgroups does not perfectly match the total number of students in components.

The subgroup correct or circular (whose possible responses include the correct answer) has only the centripetal components as dominant characteristics. The tangent components of their vectors are only prominent characteristics. These students most likely recognize the necessity of a centripetal component to acceleration for an object to change direction but do not necessarily correctly combine this knowledge with the fact that an object may be speeding up or otherwise moving along a noncircular path.

For the subgroups pure tangent and correct and tangent, the mentioned components are all dominant characteristics. Pure tangent may be an important extension to two dimensions of the commonly observed difficulty that students do not differentiate between a quantity and a change in a quantity. ${ }^{9}$ Correct and tangent is a group that we are not entirely sure how to explain as it is a response pattern we did not expect. Indeed, it is likely that had we not been using cluster analysis we would have missed this group entirely. However, since the group represents roughly $6 \%$ of our study population, there is a chance, albeit slight, that we will be able to encounter this response pattern in an interview setting in the future and thereby come to a better understanding of it.

\section{Group III: Tangent zero $(n=39)$}

The third largest group contains those students who explicitly labeled the acceleration as zero (either through text

TABLE II. Groups found by the clustering algorithm.

\begin{tabular}{|c|c|c|c|}
\hline Diagram identifier & Name & Dominant characteristics & Prominent characteristics \\
\hline I & No C arrow & No arrow at point $C$ & \\
\hline Ia & Tangent no arrow & as I plus tangent at point $B$ & \\
\hline $\mathrm{Ib}$ & CtD no arrow & as I plus connect the dots at point $B$ & \\
\hline Ic & Blanks & as I plus no arrows at point $B$ & \\
\hline II & Components & & Tangent and/or centripetal at points $B$ and $C$ \\
\hline IIa & Correct or circular & Centripetal at points $B$ and $C$ & Tangent at points $B$ and $C$ \\
\hline $\mathrm{IIb}$ & Pure tangent & Tangent at points $B$ and $C$ & \\
\hline IIc & Correct and tangent & $\begin{array}{l}\text { Tangent and centripetal at point } B \text {, tangent } \\
\text { at point } C\end{array}$ & \\
\hline III & Tangent zero & $\begin{array}{l}\text { Tangent at point } B \text { and } 0 \text { arrow at point } \\
C\end{array}$ & \\
\hline IV & Connect the dots & Connect the dots at points $B$ and $C$ & \\
\hline V & Centrifugal & & Centrifugal at points $B$ and $C$ \\
\hline VI & Curve following & Curve following at points $B$ and $C$ & \\
\hline
\end{tabular}


or symbolically) for the point with constant speed and a tangent arrow for the point with changing speed as dominant characteristics. This group may be quite similar to tangent no arrow, but was more explicit about the acceleration.

If they are, in essence, the same, then roughly $20 \%$ of the roughly 300 students fall into a category where $a=0$ at constant speed and only points in the direction of the velocity when the speed is changing. These students, who have had instruction on how to determine the direction of acceleration for an object moving in one dimension, are likely overgeneralizing their $1 \mathrm{D}$ motion response to $2 \mathrm{D}$ motion by simply ignoring the fact that the trajectory is curved. This suspicion is further supported by the fact that our percentage is roughly the same as that reported by Shaffer and McDermott ${ }^{9}$ in a much larger study.

\section{Group IV: Connect the dots $(n=29)$}

Students in the fourth group employed connect-the-dots arrows at both points.

Responses like these are similar to those of the group $C t D$ no arrow, except they also include a connect-the-dots arrow from point $C$ to $D$, on Fig. 1. These students likely have an issue of not understanding the meaning of a vector. Because a statement of "failure to understand" does not adequately describe what students are actually doing, we focus instead on the role of connecting the dots. Perhaps these students are confusing average velocity with acceleration. Such a conceptually guided response, however, seems unlikely on a pretest with students barely familiar with vectors, velocity, and acceleration. Perhaps, instead, they are unfamiliar with the visual representations and are picking out the most compelling visual attributes ${ }^{16}$ to guide their reasoning about the situation.

\section{Group V: Centrifugal $(n=16)$}

Students in the fifth group had, at one or both points, arrows that had a centrifugal component or were entirely centrifugal.

Because centrifugal arrows point "outward" from the turn, we have the possibility that students are confusing centripetal acceleration and the fictitious centrifugal force felt from inside the accelerating reference frame. Given that this group represents only about $4 \%$ of our population, we are unlikely to find many interview subjects who give this response, and thus are unlikely to find more detail on whether these students are thinking from within the accelerating reference frame.

\section{Group VI: Curve following $(n=14)$}

This group was characterized by the use of curvefollowing arrows at both points.

These responses indicate an issue of understanding what we mean by a vector drawn in the direction of the acceleration, as required in the question. "Curve-following arrows" violate several basic rules of vector drawing, the most egregious being that they are not straight arrows. It could be that the idea driving their thinking is that movement down a path is akin to acceleration. For those students who have not yet differentiated the idea of motion into velocity and acceleration, ${ }^{17,18}$ a path-following arrow might indicate no more than motion along the path. Because of the small number of such responses, we have little chance of finding these students in interviews which will clarify what they are thinking about the physics. ${ }^{19}$

\section{B. Finding themes across the groups}

An important point needs to be made when looking at issues common across many of the groups found by the cluster analysis. We give one example, to illustrate how cluster analysis shows differences in student grouping that might otherwise be clustered together: tangent no arrow, pure tangent, and tangent zero all have students drawing a tangent (and solely tangent) vector to represent the acceleration of an object which is speeding up as it goes around a curve. Their behavior for an object traveling with constant speed as it goes around a curve varies from not drawing anything (tangent no arrow) to drawing a tangent vector (pure tangent) to stating that there is no acceleration (tangent zero), but they all share a common behavior for the "more general" situation. Thus, the researcher might be tempted to group these students together into one large group.

For all that the dominant characteristics seem shared across these groups, the cluster analysis indicates that the responses are not so similar. By looking only at dominant characteristics, which exist within a single group or are shared across groups, one ignores the analysis provided by clustering all responses together. Tangent no arrow and pure tangent, for instance, do not connect to each other in the grouping of responses until almost the very end of our clustering tree (as measured from the bottom up in Fig. 4), indicating that the behaviors are fairly well separated. Tangent no arrow and tangent zero, on the other hand, join together about three steps earlier on the tree, indicating a closer relationship. However, in those three steps the joining distance goes only from about 0.17 to 0.15 , so while those three steps allow us to order the similarity between groups, the actual gain in similarity is not large.

\section{DISCUSSION}

As with any method, even one borrowed from another discipline where it is already well established, the first question that a researcher in the discipline must ask before accepting the method is "Does it work in the new domain?" Of course, one's definition of "work" affects how one answers that question. We have shown that cluster analysis can separate students into groups that can be recognized and characterized by common traits in those students' answers. Indeed, the method was able to do this without any prior knowledge on the part of the researcher of what form those groups would take (as would be needed for a more traditional method, and which would introduce bias). However, a reader might (correctly) say that the methods of cluster analysis will produce groups in any properly presented data set, even one that is generated randomly. The fact that we were able to find and describe groups is not, in and of itself, sufficient to say 
that cluster analysis methods are a viable methodology for PER. In addition we should look for two things: (1) that the defined groups reasonably make sense to a researcher, and (2) that the results of cluster analysis agree, when possible, with the results obtained by more accepted research methodologies.

For the six groups that we identified by cluster analysis, both points are addressed appropriately. To address the first point, each group can be well described by relatively few traits, and in no cases are these traits outright contradictory. Also, the groupings are reasonable; in most cases, we can infer an element of logic to the responses, even if it is not the logic that we would like our students to be using. ${ }^{20}$ The second point is addressed because several of the groups that were identified by the cluster analysis methods bear remarkable similarity to response patterns previously reported in the literature about two-dimensional kinematics. ${ }^{9}$ Further detail is required though, such as analysis of larger sets of data on identical questions and distinguishing among answer patterns between different questions. To this end, we plan a study in which data are analyzed by both the traditional methods of PER and the methods of cluster analysis. By comparing the results of each analysis we can better understand the strengths and weaknesses of each method.
Furthermore, there remains unused information within the pretests themselves that has not been discussed here. For the purposes of this preliminary study, we looked only at two points of the four that were asked about in the question and the student reasonings were not used at all. Thus while the little bit of data that we looked at does show promise, it remains to be seen if that promise will be borne out as we make our analysis more complete by adding to the SAVs dimensions for the other two points and student reasoning. What we have looked at here is only a first step.

In summary, we find that using cluster analysis leads to identifiable groups of responses on free-response questions about two-dimensional kinematics. These groups make sense to us as researchers, are consistent with previous work using more traditional analysis methods, and also provide additional information about differences between groups that were unexpected.

\section{APPENDIX: SUPPLEMENTARY AUXILIARY MATERIAL}

See separate auxiliary material for code used to perform clustering described in paper.
${ }^{1}$ L. C. McDermott and E. F. Redish, Resource Letter PER-1: Physics Education Research. Am. J. Phys. 67, 755 (1999).

${ }^{2}$ R. Duit, Bibliography_stcse: Students' and teachers' conceptions and science education, IPN-Leibniz Institute for Science Education (Kiel, Germany, 2004).

${ }^{3}$ M. C. Wittmann, in Limitations in predicting student performance on standardized tests, in Physics Education Research Conference Proceedings 2002, edited by K. C. Cummings, J. Marx, and S. Franklin (PERC Publishing, 2003), p. 9-12.

${ }^{4}$ R. E. Scherr, Modeling student thinking: An example from special relativity. Am. J. Phys. 75, 272 (2007).

${ }^{5}$ M. C. Wittmann and R. E. Scherr, in Student epistemological mode constraining researcher access to student thinking: An example from an interview on charge flow in Physics Education Research Conference Proceedings 2002, edited by K. C. Cummings, J. Marx, and S. Franklin (PERC Publishing, 2003), p. 43-46.

${ }^{6}$ M. Montenegro, G. J. I. Aubrecht, and L. Bao (unpublished).

${ }^{7} \mathrm{M}$. Montenegro (private communication).

${ }^{8}$ L. Bao and E. F. Redish, Model analysis: Representing and assessing the dynamics of student learning. Phys. Rev. ST Phys. Educ. Res. 2, 010103 (2006).

${ }^{9}$ P. S. Shaffer and L. C. McDermott, A research-based approach to improving student understanding of the vector nature of kinematical concepts. Am. J. Phys. 73, 921 (2005).

${ }^{10}$ J. R. Thompson, P. S. Shaffer, and L. C. McDermott (unpublished).

${ }^{11}$ J. R. Thompson and B. S. Ambrose (unpublished).

${ }^{12}$ P.-N. Tan, M. Steinbach, and K. Vipin, Introduction to Data Mining (Addison-Wesley, Reading, MA, 2006).

${ }^{13}$ M. de Hoon, S. Imoto, and S. Miyano, The C Clustering Library, Laboratory of DNA Information Analysis, Human Genome Center, Institute of Medical Science, University of Tokyo, 4-6-1 Shi- rokanedai, Minato-ku, Tokyo 108-8639, Japan (2007), URL http://bonsai.ims. u-tokyo.ac.jp/ mdehoon/software/ cluster/cluster.pdf

${ }^{14}$ Normally an unweighted, normalized Euclidian distance would imply that each element of the vectors represents the scalar component of the vector along a unique orthogonal dimension. Since our data are binary and the result of nonexclusionary student responses, neither of these assumptions can safely be made.

${ }^{15}$ The same threshold was used in a study carried out at Facet Innovations; Pamela Kraus (private comunication).

${ }^{16}$ A. Elby, What students' learning about representations tells us about constructivism. J. Math. Behav. 19, 481 (2000).

${ }^{17}$ D. Dykstra, Why teach kinematics? Part I, available at http:// www.boisestate.edu/physics/dykstra/WTK1.pdf (2002).

${ }^{18}$ D. Dykstra, Why teach kinematics? Part II, available at http:// www.boisestate.edu/physics/dykstra/WTK2.pdf (2002).

${ }^{19} \mathrm{We}$ have carried out preliminary interviews on the question. We have one interview in which a student did start with this response and explained why it was made. The student, however, changed the answer partway through the interview so we remain unsure if this student's reasons for using a curve following response are representative of the group as a whole, or just that particular student's idiosyncrasies.

${ }^{20} \mathrm{We}$ do not claim that all cases are expected and understood, based on correct and tangent, whose response pattern we cannot explain. We believe that continued research in this subject area will allow us to explain the logic that students with this response pattern are using and thus to strengthen our claim that the groups found by cluster analysis all have an element of logic in their makeup. 